\section{HLA-DRB1 and DQB1 genetic susceptibility to pemphigus vulgaris and pemphigus foli- aceus in Vietnamese patients}

\author{
The Bich Thanh Vuong, ${ }^{1}$ Duc Minh Do, ${ }^{2}$ \\ Phuc Thinh Ong, ${ }^{3}$ Thai Van Thanh Le ${ }^{4}$ \\ ${ }^{1}$ Department of Dermatology and \\ ${ }^{2}$ Center for Molecular Biomedicine, Ho \\ Chi Minh City University of Medicine \\ and Pharmacy at Ho Chi Minh City, Ho \\ Chi Minh City; ${ }^{3}$ Mathematical \\ Modelling Group, Centre for Tropical \\ Medicine, Oxford University Clinical \\ Research Unit, Ho Chi Minh City; \\ ${ }^{4}$ Department of Dermatology, University \\ of Medicine and Pharmacy at Ho Chi \\ Minh City; Department of Dermatology and \\ Skin Aesthetics, University Medical Center \\ HCMC, Ho Chi Minh City, Viet Nam
}

\section{Abstract \\ Pemphigus is a group of rare, life-} threatening bullous autoimmune diseases that affect the skin and mucous membranes and are associated with high morbidity and morbidity. HLA class II genes, particularly HLA-DRB1 and HLA-DQB1, play roles in pemphigus. The aim of this paper is to investigate the susceptibility of HLA class II DRB1 and DQB1 alleles in Vietnamese patients with pemphigus vulgaris (PV) or pemphigus foliaceus (PF). The study enrolled 31 participants (22 with PV, 9 with PF) with diagnoses confirmed by clinical manifestations, histopathology, and direct immunofluorescence from November 2019 to June 2020. The HLA polymorphisms were determined by Sanger sequencing. The HLA-DRB1 and HLA-DQB1 profiles of the 101 healthy individuals in the control group have been published previously. The frequencies of HLA-DRB $1 * 14$, DRB1*13:07, DRB1*04:04, DRB1*03:02, DQB1*02:02, and DQB1*05:03 were significantly higher, whereas those of DRB1*09:01, DRB1*12:02, DQB1*03:03, DQB1*05:01, and DQB1*06:01 were significantly lower, in the PV group than in the controls. The frequencies of DRB1*14:54, DRB1*13:07, and HLA-DQB1*03:02 were significantly higher in the PF group than in the controls. Alleles HLA-DRB1*14:54, DRB1*14:04, DRB1*14:03, DRB1*14:01, DRB1*14:12, DRB1*13:07, DRB1*04:04, DRB1*03:02, DQB1*02:02, and DQB1*05:03 were associated with an increased risk of $\mathrm{PV}$, whereas alleles DRB1*09:01, DRB1*12:02, DQB1*03:03, DQB1*05:01, and DQB1*06:01 might pro- tect against PV. In PF, DRB1*14:54, DRB1*13:07, and HLA-DQB1*03:02 are promising susceptibility alleles.

\section{Introduction}

Pemphigus is a group of rare, life-threatening autoimmune diseases affecting the skin and mucous membranes that are associated with high morbidity and characterised by the presence of autoantibodies against desmogleins (Dsg). ${ }^{1}$ Based on clinical and histological criteria, the disease is subcategorised into pemphigus vulgaris (PV), pemphigus foliaceus (PF), pemphigus erythematosus, pemphigus vegetans, and paraneoplastic pemphigus. ${ }^{2} \mathrm{PV}$ and $\mathrm{PF}$ are the most common clinical forms. While PF causes only skin lesions via production of anti-Dsg1 antibodies, PV causes painful blistering on both the skin and mucous membranes via production of autoantibodies against Dsg1 and Dsg3. ${ }^{3}$ Although the pathogenic mechanisms of pemphigus remain unclear, there is evidence that genetic and environmental factors, including HLA-DR, DQ genes, ${ }^{4}$ drug intake, ${ }^{5}$ and viral infections, ${ }^{6}$ contribute to its onset and progression.

HLA class II genes are highly polymorphic and encode a variety of molecules with different binding affinities to ensure a high capacity of variable antigen peptide binding to CD4+ T cells. ${ }^{7}$ HLA class II polymorphisms play an important role in the restriction of Dsg3-reactive T and B cell autoantibody production and contribute to specify Dsg-derived peptides in PV and PF. ${ }^{8,9}$ The role of HLA class II genes was confirmed in a humanised HLA DRB $1 * 04: 02$ transgenic mouse model, in which DRB1*04:02 regulated T-cell recognition of Dsg3 and induced the loss of epidermal keratinocyte adhesion, a key manifestation in PV. ${ }^{10}$ Population-based studies have reported the association between HLA class II alleles and $\mathrm{PF}$ or PV in various ethnic groups, focusing on HLA-DRB1 and DQB1. In the $\mathrm{UK}$, Saha et al. suggested that DQB ${ }^{*} 02$ is a protective allele for PV. ${ }^{11}$ Interestingly, Saha et al. found differences in the PF susceptibility alleles between white British and Indo-Asian patients, highlighting the important role of racial variation in the genetic susceptibility to disease development. ${ }^{12}$ Studies in Brazil have reported several PF susceptibility alleles, including HLADRB1*01:01, DRB1*01:02, DRB1*01:03, DRB1*04:04, DRB1*04:06, DRB1*04:10, DRB1*14:06, and DRB1*16:01, ${ }^{13,14}$ as well as the PV-associated alleles HLADRB1*04:02, DRB1*08:04, and the
Correspondence: Vuong The Bich Thanh, University of Medicine and Pharmacy at Ho Chi Minh City, 217 Hong Bang, 11 Ward, 5 District, Ho Chi Minh City, Viet Nam

Tel.: 84904425548.

E-mail: vtbthanh@ump.edu.vn

Key words: Pemphigus vulgaris, Pemphigus foliaceus, HLA-DRB1, HLA-DQB1.

Acknowledgements: The authors thank Hoang Le Gia Linh (Centre for Molecular Biomedicine, University of Medicine and Pharmacy, Ho Chi Minh City) for her technical support with the HLA typing.

Contributions: The authors contributed equally.

Conflict of interest: The authors declare no potential conflict of interest.

Funding: None.

Data availability: Due to privacy and ethical concerns, neither the data nor the source of the data can be made available.

Please cite this article as: Vuong TBT, Do $D M$, Ong PT, Le TVT. HLA-DRB1 and DQB1 genetic susceptibility to pemphigus vulgaris and pemphigus foliaceus in Vietnamese patients. Dermatol Rep 2022;14:9286.

Received for publication: 11 June 2021 Accepted for publication: 7 July 2021.

This work is licensed under a Creative Commons Attribution-NonCommercial 4.0 International License (CC BY-NC 4.0).

COCopyright: the Author(s), 2021

Licensee PAGEPress, Italy

Dermatology Reports 2022; 14:9286

doi:10.4081/dr.2021.9286

Publisher's note: All claims expressed in this article are solely those of the authors and do not necessarily represent those of their affiliated organizations, or those of the publisher, the editors and the reviewers. Any product that may be evaluated in this article or claim that may be made by its manufacturer is not guaranteed or endorsed by the publisher.

DRB $1 * 14$ group. ${ }^{15}$ A Korean study discovered significant associations of $\mathrm{PV}$ with $\mathrm{DRB} 1 * 01$ and of PF with DRB $1 * 01$, DQA $1 * 03: 02$, and DQB1*06:03. ${ }^{16}$ HLA class II alleles are considered promising genetic markers of pemphigus and are being investigated globally. ${ }^{17}$

In the Vietnamese Kinh general population, the characteristics of HLA class II alleles and haplotypes were more closely associated with Southeast Asians (Thai, Malaysians, and Indonesians) than with East Asians (Han Chinese, Japanese, and 
South Koreans). ${ }^{18}$ However, except for the study by Saha et al. that investigated IndoAsian patients living in the $\mathrm{UK},{ }^{12}$ little is known about the associations of HLADRB1 or DQB1 with PF and PV in Southeast Asians and Vietnamese. Therefore, this study investigated the PV and PF susceptibility of HLA class II DRB1 and DQB1 alleles in Vietnamese patients.

\section{Materials and Methods}

Table 1. HLA-DRB1 frequencies in PV patients compared to healthy controls.

\begin{tabular}{|c|c|c|c|c|}
\hline HLA-DRB1 & PV $(n=44)$ & Healthy individual ( $\mathrm{n}=202$ ) & OR (CI 95\%) & p \\
\hline 03:01 & $2(4.5)$ & $15(7.4)$ & $0.63(0.09-2.39)$ & 0.745 \\
\hline 03:02 & $3(6.8)$ & $0(0.0)$ & & 0.005 \\
\hline 04:01 & $0(0.0)$ & $1(0.5)$ & & 1.000 \\
\hline 04:03 & $1(2.3)$ & $3(1.5)$ & $1.67(0.06-14.79)$ & 0.548 \\
\hline 04:04 & $2(4.5)$ & $0(0.0)$ & $(2)$ & 0.031 \\
\hline 04:05 & $0(0.0)$ & $13(6.4)$ & +2 & 0.133 \\
\hline 04:06 & $0(0.0)$ & $2(1.0)$ & & 1.000 \\
\hline 07:01 & $1(2.3)$ & $6(3.0)$ & $0.85(0.03-5.32)$ & 1.000 \\
\hline 08:02 & $1(2.3)$ & $0(0.0)$ & & 0.179 \\
\hline 08:03 & $2(4.5)$ & $11(5.4)$ & $0.88(0.12-3.48)$ & 1.000 \\
\hline 08:08 & $1(2.3)$ & $0(0.0)$ & & 0.179 \\
\hline 08:12 & $0(0.0)$ & $1(0.5)$ & & 1.000 \\
\hline 09:01 & $0(0.0)$ & $27(13.4)$ & & 0.006 \\
\hline 10:01 & $0(0.0)$ & $16(7.9)$ & & 0.084 \\
\hline 11:01 & $1(2.3)$ & $5(2.5)$ & $1.02(0.04-6.83)$ & 1.000 \\
\hline 11:06 & $0(0.0)$ & $3(1.5)$ & & 1.000 \\
\hline $11: 129$ & $0(0.0)$ & $1(0.5)$ & & 1.000 \\
\hline 12:02 & $1(2.3)$ & $45(22.3)$ & $0.09(0.00-0.44)$ & 0.001 \\
\hline 13:01 & $0(0.0)$ & $1(0.5)$ & & 1.000 \\
\hline 13:02 & $0(0.0)$ & $3(1.5)$ & & 1.000 \\
\hline 13:03 & $1(2.3)$ & $0(0.0)$ & & 0.179 \\
\hline 13:07 & $2(4.5)$ & $0(0.0)$ & & 0.031 \\
\hline $13: 12$ & $0(0.0)$ & $6(3.0)$ & & 0.595 \\
\hline 14:01 & $2(4.5)$ & $0(0.0)$ & & 0.031 \\
\hline 14:03 & $2(4.5)$ & $0(0.0)$ & & 0.031 \\
\hline 14:04 & $3(6.8)$ & $1(0.5)$ & $13.31(1.51-388.34)$ & 0.019 \\
\hline 14:05 & $1(2.3)$ & $2(1.0)$ & $2.46(0.08-31.03)$ & 0.448 \\
\hline 14:06 & $1(2.3)$ & $0(0.0)$ & & 0.179 \\
\hline 14:10 & $0(0.0)$ & $1(0.5)$ & & 1.000 \\
\hline 14:12 & $2(4.5)$ & $0(0.0)$ & & 0.031 \\
\hline $14: 18$ & $0(0.0)$ & $1(0.5)$ & & 1.000 \\
\hline 14:54 & $9(20.5)$ & $3(1.5)$ & $16.22(4.49-80.15)$ & $<0.001$ \\
\hline 15:01 & $0(0.0)$ & $5(2.5)$ & & 0.589 \\
\hline 15:02 & $2(4.5)$ & $21(10.4)$ & $0.44(0.06-1.59)$ & 0.389 \\
\hline $15: 03$ & $1(2.3)$ & $0(0.0)$ & & 0.179 \\
\hline 16:01 & $1(2.3)$ & $0(0.0)$ & & 0.179 \\
\hline 16:02 & $2(4.5)$ & $9(4.5)$ & $1.08(0.15-4.48)$ & 1.000 \\
\hline
\end{tabular}

\section{Study subjects}

This cross-sectional study was conducted in Ho Chi Minh City Hospital of Dermato-Venereology from November 2019 to June 2020. Patients with a confirmed diagnosis of pemphigus based on clinical manifestations, histopathology, and direct immunofluorescence showing IgG at intercellular borders in the epidermis were enrolled. Other eligibility criteria were Vietnamese Kinh descent and age of at least 18 years. Exclusion criteria were a history allogeneic stem cell transplantation and other coexisting autoimmune diseases. All subjects underwent a thorough physical and laboratory examination by a research doctor. The control group consisted of 101 healthy Kinh Vietnamese, the HLA-DRB1 and DQB1 profiles of which have been published. ${ }^{18}$

\section{DNA extraction}

Venous blood ( $2 \mathrm{ml}$ ) was collected from each patient into an EDTA-K2 anticoagulant tube. Genomic DNA was extracted from whole blood using the GeneJET Whole Blood Genomic DNA Purification Mini Kit (Thermo Scientific, USA), according to the manufacturer's protocol. 


\section{Library preparation}

PCR amplification and target gene sequencing were performed using primers complementary to HLA-DRB1 (acc. no. NG_002392) and HLA-DQB1 (acc. no. NG_02922) sequences, obtained from $\mathrm{NCB}$ or Lazaro et al. ${ }^{19}$

\section{Sequencing}

Sanger sequencing was performed using POP-7 ${ }^{\mathrm{TM}}$ Polymer and a $50 \mathrm{~cm}$ capillary array on the ABI 3500 Genetic Analyser (Applied Biosystems, USA). The sequences were subsequently analysed using the CLC Main Workbench and compared using BLAST in EBI software to identify the HLA-DRB1 and DQB1 alleles of the participants.

\section{Statistical analysis}

Continuous data are presented as the mean \pm standard deviation or median with interquartile range according to the normality of the data. Categorical data are described as the frequency and percentage. Quantitative variables were analysed using Student's $t$-test. Fisher's exact test was used to compare allele frequencies between the pemphigus subtypes. A two-sided $\mathrm{p}<0.05$ was considered statistically significant. All analyses were conducted using $\mathrm{R}$ ver. 4.0.2 (Vienna, Austria).

\section{Ethics}

This study was approved by the Biomedical Research Ethics Review Committee of the University of Medicine and Pharmacy, Ho Chi Minh City, Vietnam (approval number: 593/DHYD-HDDD, November 4, 2019). Informed consent was received from all participants.

\section{Results}

In the $22 \mathrm{PV}$ and $9 \mathrm{PF}$ patients enrolled in this study, 23 and 10 HLA-DRB1 alleles and 9 and 7 HLA-DQB1 alleles were detected, respectively.

\section{HLA-DRB1 profile in the PV group}

HLA-DRB1*14:54 and DRB1*14:04 were the two alleles most strongly associated with PV susceptibility [OR $=16.22$ $(4.49-80.15)$ and $\mathrm{OR}=13.31(1.51-$ 388.34), respectively]. Other susceptibility alleles detected in the PV patients, but not in healthy controls, were DRB1*03:02 $(p=0.005), \quad D R B 1 * 04: 04 \quad(p=0.031)$, DRB1*13:07 $(\mathrm{p}=0.031), \quad \mathrm{DRB} 1 * 14: 01$ $(p=0.031), \quad D R B 1 * 14: 03 \quad(p=0.031)$, and $\mathrm{DRB} 1 * 14: 12(\mathrm{p}=0.031)$. The frequencies of DRB1*09:01 $(\mathrm{p}=0.006)$ and DRB1*12:02 $(p=0.001)$ were significantly higher in the control group (Table 1).

\section{HLA-DQB1 profile in the PV group}

Regarding HLA-DQB1 polymorphisms, the DQB1*02:02 $(\mathrm{p}=0.011)$ and DQB1*05:03 $(\mathrm{p}<0.001)$ alleles were more frequent $[\mathrm{OR}=6.23(1.52-27.34)$ and 12.65 (4.81-36.36), respectively], whereas DQB1*03:03 $(\mathrm{p}=0.036), \quad \mathrm{DQB} 1 * 05: 01$ $(\mathrm{p}=0.011)$, and $\mathrm{DQB} 1 * 06: 01 \quad(\mathrm{p}=0.048)$ were less frequent, in the $\mathrm{PV}$ patients than healthy controls (Table 2).

\section{HLA-DRB1 profile in the PF group}

In the HLA-DRB1 region, the frequencies of DRB1*14:54 [OR = $30.99(7.05$ 172.21), $\quad \mathrm{p}<0.001]$ and DRB1*13:07 $(\mathrm{p}=0.006)$ were significantly higher in the PF group than controls (Table 3).

\section{HLA-DQB1 profile in the PF group}

HLA-DQB $1 * 03: 02$ was significantly more frequent in the PF patients than controls $[\mathrm{OR}=14.68(3.55-61.44), \mathrm{p}<0.001]$ (Table 4).

\section{Discussion}

HLA genes are reported to be associated with the occurrence and development of pemphigus. ${ }^{20}$ HLA class II antigens are typically expressed on the surface of active $\mathrm{T}$ cells, which are believed to play a major role in $T$ cell activation and antigen recognition. ${ }^{21}$ Importantly, studies have highlighted the importance of racial variation in the genetic susceptibility to pemphigus, ${ }^{12}$ indicating the need for profiling and investigation of the genetic factors of the disease among different ethnic groups and populations. However, few studies have examined pemphigus patients living in Southeast Asia. This is the first study to identify HLADRB1 and HLA-DQB1 susceptibility alle-

Table 2. HLA-DQB1 frequencies in PV patients compared to healthy controls.

\begin{tabular}{|c|c|c|c|c|}
\hline HLA-DQB 1 & PV $(n=44)$ & Healthy individual ( $\mathrm{n}=202$ ) & OR (CI 95\%) & p \\
\hline 02:01 & $1(2.3)$ & $14(6.9)$ & $0.35(0.01-1.85)$ & 0.483 \\
\hline 02:02 & $5(11.4)$ & $4(2.0)$ & $6.23(1.52-27.34)$ & 0.011 \\
\hline 03:01 & $13(29.5)$ & $58(28.7)$ & $1.05(0.49-2.11)$ & 1.000 \\
\hline 03:02 & $2(4.5)$ & $5(2.5)$ & $1.95(0.24-9.83)$ & 0.612 \\
\hline 03:03 & $1(2.3)$ & $27(13.4)$ & $0.17(0.01-0.84)$ & 0.036 \\
\hline 03:05 & $0(0.0)$ & $1(0.5)$ & & 1.000 \\
\hline 04:01 & $0(0.0)$ & $10(5.0)$ & & 0.216 \\
\hline 04:02 & $0(0.0)$ & $2(1.0)$ & & 1.000 \\
\hline 05:01 & $0(0.0)$ & $25(12.4)$ & & 0.011 \\
\hline 05:02 & $8(18.2)$ & $23(11.4)$ & $1.74(0.68-4.10)$ & 0.217 \\
\hline 05:03 & $14(31.8)$ & $7(3.5)$ & $12.65(4.81-36.36)$ & $<0.001$ \\
\hline $05: 10$ & $0(0.0)$ & $1(0.5)$ & & 1.000 \\
\hline 05:18 & $0(0.0)$ & $2(1.0)$ & & 1.000 \\
\hline 06:01 & $0(0.0)$ & $17(8.4)$ & & 0.048 \\
\hline 06:02 & $0(0.0)$ & $2(1.0)$ & & 1.000 \\
\hline 06:03 & $0(0.0)$ & $1(0.5)$ & & 1.000 \\
\hline 06:04 & $0(0.0)$ & $1(0.5)$ & & 1.000 \\
\hline 06:09 & $0(0.0)$ & $2(1.0)$ & & 1.000 \\
\hline
\end{tabular}


Table 3. HLA-DRB1 frequencies in PF patients compared to healthy controls.

\begin{tabular}{|c|c|c|c|c|}
\hline HLA-DRB1 & PF $(n=18)$ & Healthy individual ( $\mathrm{n}=202$ ) & OR (CI 95\%) & p \\
\hline 03:01 & $2(11.1)$ & $15(7.4)$ & $1.64(0.22-6.68)$ & 0.636 \\
\hline 03:02 & $1(5.6)$ & $0(0.0)$ & & 0.082 \\
\hline 04:01 & $0(0.0)$ & $1(0.5)$ & & 1.000 \\
\hline 04:03 & $2(11.1)$ & $3(1.5)$ & $8.30(0.91-58.38)$ & 0.055 \\
\hline 04:05 & $1(5.6)$ & $13(6.4)$ & $0.96(0.04-5.38)$ & 1.000 \\
\hline 04:06 & $1(5.6)$ & $2(1.0)$ & $6.14(0.19-79.40)$ & 0.227 \\
\hline 04:07 & $1(5.6)$ & $0(0.0)$ & & 0.082 \\
\hline 07:01 & $1(5.6)$ & $6(3.0)$ & $2.12(0.08-14.00)$ & 0.455 \\
\hline 08:03 & $0(0.0)$ & $11(5.4)$ & & 0.606 \\
\hline 08:12 & $0(0.0)$ & $1(0.5)$ & & 1.000 \\
\hline 09:01 & $0(0.0)$ & $27(13.4)$ & & 0.138 \\
\hline 10:01 & $0(0.0)$ & $16(7.9)$ & & 0.373 \\
\hline 11:01 & $0(0.0)$ & $5(2.5)$ & & 1.000 \\
\hline 11:06 & $0(0.0)$ & $3(1.5)$ & & 1.000 \\
\hline $11: 129$ & $0(0.0)$ & $1(0.5)$ & & 1.000 \\
\hline 12:02 & $1(5.6)$ & $45(22.3)$ & $0.23(0.01-1.19)$ & 0.131 \\
\hline 13:01 & $0(0.0)$ & $1(0.5)$ & & 1.000 \\
\hline 13:02 & $0(0.0)$ & $3(1.5)$ & 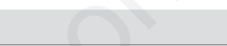 & 1.000 \\
\hline 13:07 & $2(11.1)$ & $0(0.0)$ & $\omega$ & 0.006 \\
\hline 13:12 & $0(0.0)$ & $6(3.0)$ & & 1.000 \\
\hline 14:04 & $0(0.0)$ & $1(0.5)$ & & 1.000 \\
\hline 14:05 & $0(0.0)$ & $2(1.0)$ & & 1.000 \\
\hline 14:10 & $0(0.0)$ & $1(0.5)$ & & 1.000 \\
\hline 14:18 & $0(0.0)$ & $1(0.5)$ & & 1.000 \\
\hline 14:54 & $6(33.3)$ & $3(1.5)$ & $30.99(7.05-172.21)$ & $<0.001$ \\
\hline 15:01 & $0(0.0)$ & $5(2.5)$ & & 1.000 \\
\hline 15:02 & $0(0.0)$ & $21(10.4)$ & & 0.229 \\
\hline 16:02 & $0(0.0)$ & $9(4.5)$ & & 1.000 \\
\hline
\end{tabular}

Table 4. HLA-DQB1 frequencies in PF patients compared to healthy controls.

\begin{tabular}{|c|c|c|c|c|}
\hline HLA-DRB1 & PF $(n=18)$ & Healthy individual $(\mathrm{n}=202)$ & OR (CI 95\%) & p \\
\hline 02:01 & $1(5.6)$ & $14(6.9)$ & $0.89(0.04-4.92)$ & 1.000 \\
\hline 02:02 & $0(0.0)$ & $4(2.0)$ & & 1.000 \\
\hline 03:01 & $5(27.8)$ & 58 (28.7) & $0.97(0.29-2.74)$ & 1.000 \\
\hline 03:02 & $5(27.8)$ & $5(2.5)$ & $14.68(3.55-61.44)$ & $<0.001$ \\
\hline 03:03 & $0(0.0)$ & 27 (13.4) & & 0.138 \\
\hline 03:05 & $0(0.0)$ & $1(0.5)$ & & 1.000 \\
\hline 03:09 & $1(5.6)$ & $0(0.0)$ & & 0.082 \\
\hline 04:01 & $0(0.0)$ & $10(5.0)$ & & 1.000 \\
\hline 04:02 & $0(0.0)$ & $2(1.0)$ & & 1.000 \\
\hline 05:01 & $1(5.6)$ & $25(12.4)$ & $0.47(0.02-2.47)$ & 0.703 \\
\hline 05:02 & $4(22.2)$ & 23 (11.4) & $2.27(0.58-7.06)$ & 0.249 \\
\hline 05:03 & $1(5.6)$ & $7(3.5)$ & $1.82(0.07-11.47)$ & 0.501 \\
\hline $05: 10$ & $0(0.0)$ & $1(0.5)$ & & 1.000 \\
\hline 05:18 & $0(0.0)$ & $2(1.0)$ & & 1.000 \\
\hline 06:01 & $0(0.0)$ & $17(8.4)$ & & 0.372 \\
\hline 06:02 & $0(0.0)$ & $2(1.0)$ & & 1.000 \\
\hline 06:03 & $0(0.0)$ & $1(0.5)$ & & 1.000 \\
\hline 06:04 & $0(0.0)$ & $1(0.5)$ & & 1.000 \\
\hline 06:09 & $0(0.0)$ & $2(1.0)$ & & 1.000 \\
\hline
\end{tabular}


les in Kinh Vietnamese PV and PF patients and should contribute to the knowledge on pemphigus genetic factors in Vietnamese and Southeast Asians.

The frequencies of the HLADRB1*14:54, DRB1*14:04, DRB1*14:03, DRB1*14:01, DRB1*14:12, DRB1*13:07, DRB1*04:04, and DRB1*03:02 alleles were significantly higher in the PV group than healthy controls among Kinh Vietnamese. Overall, the DRB1*14 allele group is associated with susceptibility to $\mathrm{PV}$, which is similar to the findings of Saha et al. in the UK, ${ }^{22}$ Glorio et al. in Argentina, ${ }^{21}$ Gil et al. in Brazil, ${ }^{23}$ Párnická et al. in Slovakia, ${ }^{24}$ and Porro et al. in India. ${ }^{25}$ In China, Geng et al. reported several PV-associated alleles similar to our findings, specifically the DRB ${ }^{*} 14$ allele group (DRB1*14:01, DRB1*14:04, DRB1*14:05, DRB1*14:07, and DRB1*14:08). ${ }^{26}$ Concordant results from multiple genetic analyses worldwide, including ours, suggest that the DRB $1 * 14$ allele group is a promising biomarker of PV that is independent of race or ethnicity.

The association between the DRB1*14:01 allele and PV has been reported worldwide, such as by Lombardi et al. in Italy, ${ }^{27}$ Loiseau et al. in France, ${ }^{9}$ and Glorio et al. in Argentina. ${ }^{21}$ This association is supported by molecular evidence that the DRB1*14:01 allele restricts the T-cell responses to Dsg3, the specific PV antigen that plays an essential role in cell-cell adhesion among keratinocytes. ${ }^{4}$ Note that DRB1*14:54 was previously considered to be DRB $1 * 14: 01$ and was only identified as a new allele in 2005 by the World Health Organisation Nomenclature Committee. ${ }^{28}$ It is possible that the susceptibility allele DRB1*14:01 evaluated in studies conducted before 2005 is actually DRB1*14:54. ${ }^{17,22}$ Consistent with our findings, many studies worldwide have suggested that DRB1*14:54 is a susceptibility allele for $\mathrm{PV}$, such as Saha et al. in the UK and Párnická et al. in Slovakia. ${ }^{11,24}$ Other studies reported a relationship of the DRB $1 * 04: 04$ and DRB1*14:04 alleles with PV. Párnická et al. showed that DRB1*04:04 [OR = $30.57(1.67-559.40)]$ and DRB1*14:04 $[\mathrm{OR}=24.71(1.32-464.28)]$ significantly increase the risk of PV. ${ }^{24}$ In China, Zhang et al. confirmed the association between DRB1*14:04 and PV using an Affymetrix array. $^{29}$

Regarding HLA-DQB1, the frequency of the DQB $1 * 05: 03$ allele was significantly higher in our PV patients compared with the controls, suggesting that DQB $1 * 05: 03$ is a susceptibility allele. This is consistent with Saha et al., who reported that DQB $1 * 05: 03$ significantly increased the risk of $\mathrm{PV}$ among British Indonesians $[\mathrm{OR}=6.93$ (3.03-16.48)] and white British $[\mathrm{OR}=6.18$ (3.07-12.57)]. ${ }^{11}$ Glorio et al. in Argentina $[\mathrm{RR}=8.02(3.03-21.48)]$ and Párnická et al. in Slovakia $[\mathrm{OR}=10.40$ (3.99-27.10); $\mathrm{p}<0.0001]$ reported a similar association between DQB1*05:03 and PV compared with controls. ${ }^{21,24}$ A meta-analysis by Li et al. pooled data from 18 studies and confirmed the association between the DQB $1 * 05: 03$ allele and PV $[\mathrm{OR}=10.02$ (5.41-18.55)]. ${ }^{8}$ Due to the consistent association of this allele with PV in studies worldwide, we suggest that the DQB $1 * 05: 03$ allele should be regarded as a global genetic factor for PV. Interestingly, none of our PV patients carried the HLADQB $1 * 06$ allele, while the healthy controls had several HLA-DQB1*06 alleles, including DQB1*06:01 (8.4\%), DQB1*06:02 (1.0\%), DQB1*06:03 (0.5\%), DQB1*06:04 $(0.5 \%)$, and $\mathrm{DQB} 1 * 06: 09(1.0 \%)$. This implies that DQB1*06:01 protects against PV. Recently, Dere et al. derived the same conclusion about the HLA-DQB1*06 allele as a protective factor against $\mathrm{PV}$ in Turkey. ${ }^{30}$

Similar to findings in the PV group, DRB1*14:54 was a susceptibility allele for $\mathrm{PF}[\mathrm{OR}=30.99$ (7.05-172.21)]. There was no difference in the frequency of this allele between the PV and PF groups $(p=0.334)$, implying that it is not specific to any subtype. This is in contrast to the study of Saha et al., who found that the DRB1*14:54 allele was associated with PV in Caucasians, ${ }^{11}$ but not with PF in either ethnic group (British and Indo-Asian patients). ${ }^{12}$ However, Rovesti et al. recently reported a 19-year-old European female who carried the DRB1*14:54 allele and presented with both $\mathrm{PV}$ and PF. ${ }^{30}$ Transitions from PV to PF and vice versa have been reported. However, according to Rovesti et al., this might be an extremely rare situation in which both conditions present simultaneously; only four such cases have been reported since $1998 .{ }^{31}$ The case supports our findings that the DRB1*14:54 allele is not specific to either PV or PF. Vietnamese patients with PF had a significantly higher DQB1*03:02 allele frequency compared with the controls. This result is similar to that of Zhang et al., confirming that the $\mathrm{DQB} 1{ }^{*} 03: 02$ allele is specific to $\mathrm{PF}$ in Chinese patients. ${ }^{29}$ Abida et al. investigated 90 Tunisian PF patients and found that the DQB $1 * 03: 02$ allele was statistically associated with PF. ${ }^{32}$

\section{Limitations}

Due to the rarity of this disease, we could only recruit a relatively small number of participants, and this our study might be subject to selection bias. Multicentre studies with larger samples are needed to confirm our results and obtain more comprehensive data on pemphigus patients.

\section{Conclusions}

The HLA-DRB1*14:54, DRB1*14:04, DRB1*14:03, DRB1*14:01, DRB1*14:12, DRB1*13:07, DRB1*04:04, DRB1*03:02, $\mathrm{DQB} 1 * 02: 02$, and DQB1*05:03 alleles were associated with an increased risk of PV, while DRB1*09:01, DRB1*12:02, DQB1*03:03, DQB1*05:01, and DQB1*06:01 appeared to have protective effects on PV. For PF, DRB1*14:54, DRB1*13:07, and HLA-DQB1*03:02 were found to be promising susceptibility alleles.

\section{References}

1. Pollmann R, Schmidt T, Eming R, Hertl $M$. Pemphigus: a comprehensive review on pathogenesis, clinical presentation and novel therapeutic approaches. Clin Rev Allergy Immunol 2018;54:1-25.

2. Ruocco V, Ruocco E, Lo Schiavo A, et al. Pemphigus: Etiology, pathogenesis, and inducing or triggering factors: Facts and controversies. Clin Dermatol 2013;31:374-81.

3. Amagai M, Stanley JR. Desmoglein as a target in skin disease and beyond. J Invest Dermatol 2012;132:776-84.

4. Shams S, Amirzargar AA, Yousefi M, et al. HLA class II (DRB, DQA1 and DQB1) allele and haplotype frequencies in the patients with pemphigus vulgaris. J Clin Immunol 2009;29:175-9.

5. Brenner S, Tur E, Shapiro J, et al. Pemphigus vulgaris: environmental factors. Occupational, behavioral, medical, and qualitative food frequency questionnaire. Int J Dermatol 2001;40:5629.

6. Esmaili N, Hallaji Z, Abedini R, et al. Pemphigus vulgaris and herpesviruses: is there any relationship? Int J Dermatol 2010;49:1261-5.

7. Dey-Rao R, Seiffert-Sinha K, Sinha AA. Genome-wide expression analysis suggests unique disease-promoting and disease-preventing signatures in Pemphigus vulgaris. Genes Immunity 2013;14:487-99.

8. Li S, Zhang Q, Wang $\mathrm{P}$, et al. Association between HLA-DQB1 polymorphisms and pemphigus vulgaris: A meta-analysis. Immunol Investigat 2018;47:101-12.

9. Loiseau P, Lecleach L, Prost C, et al. HLA class II polymorphism contributes 
to specify desmoglein derived peptides in pemphigus vulgaris and pemphigus foliaceus. J Autoimmun 2000;15:6773.

10. Eming R, Hennerici T, Bäcklund J, et al. Pathogenic IgG antibodies against desmoglein 3 in pemphigus vulgaris are regulated by HLA-DRB1*04:02restricted T cells. J Immunol 2014;193: 4391.

11. Saha M, Harman K, Mortimer NJ, et al. Pemphigus vulgaris in white Europeans is linked with HLA class II allele HLA DRB1* 1454 but not DRB1* 1401. J Investig Dermatol 2010;130:311-4.

12. Saha M, Harman K, Mortimer NJ, et al. Sporadic pemphigus foliaceus and class II human leucocyte antigen allele associations in the white British and IndoAsian populations in the UK. Clin Exp Dermatol 2019;44:290-4.

13. Cerna M, Fernandez $\square$ Viña M, Friedman H, et al. Genetic markers for susceptibility to endemic Brazilian pemphigus foliaceus (fogo selvagem) in Xavante Indians. Tissue Antigens 1993;42:138-40.

14. Pavoni DP, Roxo VMMS, Marquart Filho A, Petzl-Erler ML. Dissecting the associations of endemic pemphigus foliaceus (fogo selvagem) with HLADRB1 alleles and genotypes. Genes Immunity 2003;4:110-6.

15. Weber R, Monteiro F, Preuhs-Filho G, et al. HLA-DRB1*04:02, DRB1*08:04 and $\mathrm{DRB} 1 * 14$ alleles associated to pemphigus vulgaris in southeastern Brazilian population. Tissue Antigens 2011;78:92-3.

16. Lee CW, Yang HY, Kim SC, et al. HLA class II allele associations in Korean patients with pemphigus. Dermatology 1998;197:349-52.

17. Svecova D, Parnicka Z, Pastyrikova L, et al. HLA DRB1* and DQB1* alleles are associated with disease severity in patients with pemphigus vulgaris. Int $\mathrm{J}$ Dermatol 2015;54:168-73.

18. Do MD, Le LGH, Nguyen VT, et al. High-resolution HLA typing of HLA-A, -B, -C, -DRB1, and -DQB1 in Kinh Vietnamese by using next-generation sequencing. Front Genet 2020;11:383.

19. Lazaro A, Tu B, Yang R, et al. Human leukocyte antigen (HLA) typing by DNA sequencing. Methods Mol Biol Clifton NJ 2013;1034:161-95.

20. Tron F, Gilbert D, Mouquet H, et al. Genetic factors in pemphigus. J Autoimmun 2005;24:319-28.

21. Glorio R, Rodriguez Costa G, Haas R, et al. HLA haplotypes and class II molecular alleles in Argentinian patients with pemphigus vulgaris. J Cutan Med Surg 2002;6:422-6.

22. Saha M, Bhogal B, Black MM, et al. Prognostic factors in pemphigus vulgaris and pemphigus foliaceus. $\mathrm{Br} \mathrm{J}$ Dermatol 2014;170:116-22.

23. Gil JM, Weber R, Rosales CB, et al. Study of the association between human leukocyte antigens (HLA) and pemphigus vulgaris in Brazilian patients. Int $\mathrm{J}$ Dermatol 2017;56:557-62.

24. Párnická Z, Švecová D, Javor J, et al. High susceptibility to pemphigus vulgaris due to HLA-DRB1*14:54 in the Slovak population. Int J Immunogenet 2013;40:471-5.

25. Porro AM, Caetano LdVN, Maehara LdSN, Enokihara MMdS. Non-classical forms of pemphigus: pemphigus her- petiformis, IgA pemphigus, paraneoplastic pemphigus and IgG/IgA pemphigus. Anais Brasileiros Dermatologia 2014;89:96-106.

26. Geng L, Wang Y, Zhai N, et al. Association between pemphigus vulgaris and human leukocyte antigen in Han nation of northeast China. Chinese Med Sci J 2005;20:166-70.

27. Lombardi ML, Mercuro O, Pirozzi G, et al. Common human leukocyte antigen alleles in pemphigus vulgaris and pemphigus foliaceus Italian patients. J Invest Dermatol 1999;113:107-10.

28. Horn PA, Albis-Camps M, Verboom M, et al. The nature of diversity of HLADRB1 exon 3. Tissue Antigens 2007;70:335-7.

29. Zhang SY, Zhou XY, Zhou XL, et al. Subtype-specific inherited predisposition to pemphigus in the Chinese population. Br J Dermatol 2019;180:828-35.

30. Dere G, Yavuz IH, Ozaydın Yavuz G, et al. Assessment of HLA-A, HLA-DR, and HLA-DQ alleles in patients with pemphigus vulgaris from eastern of Turkey. J Cosmet Dermatol 2020;19:2432-7.

31. Rovesti M, Pierobon E, Vaschieri C, et al. Case of a severe vulgaris and foliaceus pemphigus in a young patient treated with rituximab, with subsequent development of chronic urticaria. Dermatol Ther 2020;33:e13665.

32. Abida O, Zitouni M, Kallel-Sellami M, et al. Tunisian endemic pemphigus foliaceus is associated with the HLA-DR3 gene: anti-desmoglein 1 antibody-positive healthy subjects bear protective alleles. Br J Dermatol 2009;161:522-7. 